\title{
FUNDACIÓN ALADINA: UN GIRO MÁS AL CÁNCER PEDIÁTRICO
}

\author{
ALADINA FOUNDATION: A TWIST TO PEDIATRIC CANCER
}

Valeria Moriconi

Fundación Aladina

\section{EL CÁNCER EN NIÑOS Y ADOLESCENTES}

Cualquier enfermedad determina en la persona una condición de profunda crisis y sufrimiento. El sufrimiento físico, la experiencia de límite y la realidad de ser "diferente" interrumpen el desarrollo existencial normal, poniendo a prueba la relación con uno mismo, con el cuerpo y con el entorno. La hospitalización es un ejemplo directo de cómo la condición de enfermo es algo complejo, no solo el paciente se tiene que alejar de su casa, también de sus afectos, amigos, intereses, alterando el sentido del tiempo, que ahora se modifica para seguir las reglas y los ritmos de otra realidad ${ }^{(1)}$. Este cambio tiene significados emotivos intensos con posibles consecuencias que pueden perdurar en el tiempo también cuando se vuelve a casa $^{(2)}$. Cuando el niño enferma y requiere ser hospitalizado, surge el difícil problema que supone extraerlo de su entorno habitual y de sus mecanismos de seguridad, como son su casa y su familia, para introducirlo en un medio totalmente desconocido para él, agresivo y hostil. La hospitalización es conocida como una experiencia traumática tanto para el niño como para su familia, y mucho más si se trata de una enfermedad oncológica, donde los procedimientos para combatirla forman un conjunto de actuaciones que el niño puede vivir como extrañas y amenazantes ${ }^{(3)}$. Se rompe el equilibrio tanto para el adulto como para el niño. Los niños y adolescentes pasan por las mismas etapas que los adultos frente a una enfermedad: rabia, negación, regateo, depresión y aceptación. La mayor dificultad está en escucharlos y comprenderlos, ya que se sienten solos y necesitan calor humano(4).

Lo que no tenemos que olvidar cuando hablamos de cáncer infantil es que el niño enfermo, ante todo, es un niño; por este motivo, es muy importante hablar de cuidado en todos los aspectos de la vida del paciente. No solo tenemos que cuidar su salud física, tenemos que cuidar su salud psíquica, emocional, espiritual, sus necesidades como niño y como adolescente, sus derechos y sus deberes.

Hoy en día la Oncología Pediátrica ha evolucionado mucho como medio y sobre todo como forma de concebir la salud del paciente; A este respecto, el punto clave es preservar indemne la infancia y la adolescencia lo más posible.

Este es el marco de cuidado integral del niño y del adolescente con cáncer en el que se centra la Fundación Aladina.

\section{Correspondencia:}




\section{ORIGEN Y MARCO DE INSCRIPCIÓN DE LA FUNDACIÓN ALADINA}

La Fundación Aladina nace en el 2005 con el objetivo de ayudar a niños y adolescentes enfermos de cáncer y a sus familias. Esta iniciativa surge de la experiencia personal de Paco Arango, que a lo largo de 5 años acudió semanalmente como voluntario al Hospital Infantil Universitario Niño Jesús, tras lo cual decidió involucrarse más a fondo y crear la "Fundación Aladina".

Lo que vio nuestro Fundador es que en la Oncología pediátrica se estaban dando pasos de gigantes para la acomodación de los niños a la situación hospitalaria. Estaba aumentando la capacidad de los niños de adaptarse positivamente a la adversidad; la capacidad para sobreponerse y funcionar con normalidad, para mantener un funcionamiento físico y psicológico saludable frente al estrés que comporta un diagnostico de cáncer; la habilidad para ser transformado positivamente por estas situaciones. En una palabra, había una capacidad de resiliencia más elevada ${ }^{(5)}$.

Sin embargo, había un gran número de pacientes que no se podía descuidar: los adolescentes.

Sabemos perfectamente que la etapa de la adolescencia es un periodo crucial y delicado para el desarrollo de una persona; en esta etapa de la vida asistimos a grandes transformaciones físicas, intelectuales y sociales. El individuo lucha por su propia identidad y autoimagen, por su independencia, por adaptarse a su sexualidad y a su cuerpo en continuo cambio, por establecer relaciones sociales y por planificar su futuro. Si en este momento de gran energía y de gran apertura hacia el futuro se interpone un diagnostico de cáncer, la persona se hace más vulnerable y frágil(6).

Los adolescentes viven su enfermedad como una amenaza a su independencia, como una pérdida de autocontrol y consecuentemente como una dependencia forza- da hacia los padres y el personal sanitario; se producen así frustración e impotencia. Cuando la pertenencia a un grupo de pares y la uniformidad a ello se hacen necesidad, la situación de enfermedad se configura como una condición de diversidad exasperada, no tanto a nivel físico, sino sobre todo a nivel emocional, sin posibilidad de encuentro y de interacción con los demás. Aunque hoy es raro encontrar situaciones de verdadera exclusión y aislamiento de la vida social, todavía persisten sentimientos de soledad e incomunicabilidad que implican este sentimiento de diferenciación respecto a los demás ${ }^{(7)}$. Nacen preocupaciones y dudas, sentimientos de miedo y ansiedad pero difícilmente todo esto es expresado verbalmente, sobretodo con adultos.

Consciente de estas dificultades, la Fundación Aladina decidió abrir la Sala de Adolescentes en la Unidad de Oncología del Hospital Universitario Niño Jesús de Madrid. Esta sala es un espacio exclusivo para los jóvenes, lejos de padres, médicos y del personal sanitario. En ella los adolescentes se pueden relajar, reclamar su espacio personal y relacionarse con otros chicos de su edad en sus mismas condiciones. La sala está equipada con consolas de videojuegos, ordenadores y todo tipo de material artístico y audiovisual, además en ella se llevan a cabo periódicamente talleres y actividades enfocados a esta franja de edad.

Gracias a la colaboración de voluntarios atentos y eficientes podemos ofrecer a nuestros adolescentes un abanico amplio, siempre nuevo y actual, de actividades. Nuestra atención está puesta en facilitar herramientas de desarrollo y creatividad a los chicos para superar esta fase de sus vidas y para encontrar salida a la monotonía de la situación hospitalaria.

\section{OBJETIVOS DE LA INTERVENCIÓN}

Los objetivos específicos que queremos alcanzar son: combatir el aislamiento so- 
cial y los sentimientos de vergüenza; aumentar la comunicación y la cooperación; reforzar la identidad personal y la imagen corporal.

El punto de partida general es el empleo del juego de forma terapéutica.

El juego como forma de expresión global del adolescente, de sus sentimientos, de sus temores, de sus deseos, de su realidad, y como fenómeno universal que facilita el crecimiento y conduce a las relaciones grupales.

Muchos estudios subrayan la importancia del juego en el ambiente hospitalario, puesto que permite a los pacientes encontrar un espacio, dentro del hospital, en el que sacar y externalizar vivencias y sensaciones sentidas como únicas, ajenas, dominadas de expectativas fantasiosas inaguantables ${ }^{(8)}$. Tanto que la ausencia de ocasiones de juego puede acentuar comportamientos de cierre de los pacientes y retrasar o bloquear el proceso de adaptación y de superación del evento crítico $^{(9)}$.

En la Sala de Adolescentes los chicos deciden libremente con qué jugar, así descubrimos como una lucha al "Tekken" puede ayudar a canalizar la rabia de un mal día; un partido al "FIFA 11" favorece la integración o el nacimiento de un grupo; un juego de la "Xbox Kinect" aumenta el movimiento físico; un quiz despierta la competitividad y la actividad cerebral; un karaoke es útil como medio para bajar los niveles de vergüenza. Cualquier juego en Aladina es usado como modo de comunicación consigo mismo y con los demás.

Además del juego libre, la Fundación organiza periódicamente talleres con diferentes fines. Se han Ilevado a cabo de musicoterapia para encontrar diversión, consuelo físico y psíquico y otro medio de expresión de sentimientos y pensamientos $^{(10)}$; talleres de cine para aumentar la atención y la comunicación a través del intercambio de ideas y opiniones; talleres para crear un blog con la convicción de que el uso consciente e inteligente de Internet es de gran ayuda para la socialización y para poder compartir experiencias y herramientas de superación; talleres de fotografía para dar voz de forma creativa y diferente a las emociones; talleres de manualidades como "Crea tu propia joya" y "Customiza tu ropa" fomentan la creatividad y la cohesión entre los chicos.

Gracias a este espacio, físico y psicológico de relación entre pares, el aislamiento social, la regresión y la sensación de diversidad disminuyen notablemente. Los chicos finalmente encuentran entre actividad y actividad la posibilidad de hablar de lo que les está pasando, de sus miedos y de sus pensamientos, expresan emociones y esperanzas; encuentran un espacio seguro y un ambiente abierto a la comunicación. Los adolescentes se muestran y dejan salir sus sentimientos solo cuando se sienten cómodos y estas condiciones se dan solo gracias al desarrollo de una relación de contacto genuino y sincero.

Teniendo presentes las intervenciones psicológicas que favorecen la adaptación psicosocial del adolescente con cáncer recomendadas por Die Trill(11) queremos facilitar la expresión afectiva del adolescente permitiendo manifestaciones de ira, tristeza y preocupación; facilitamos la discusión acerca de estos sentimientos, normalizándolos y encauzándolos adecuadamente.

Gracias a un equipo de voluntarios fuerte y variado en edades e intereses, los adolescentes pueden volver a crear redes sociales y grupos de amistades. El grupo de amigos es el pilar en que el adolescente se apoya y el núcleo que le hace sentir vivo y avanzar hacia el futuro.

Sabiendo que están viviendo la misma experiencia se sienten libres para hablar de tratamientos médicos y secuelas físicas con naturalidad y sin filtros, haciendo preguntas delicadas con el tono "desaliñado" típico de la adolescencia. Encuentran la forma de 
ironizar sobre su enfermedad y dar consejos o "trucos" a otros compañeros cuando los necesitan. Nacen espontáneamente "cadenas de solidaridad donde uno levanta al otro cuando está mal". La canalización positiva y constructiva de las emociones vuelve a encontrar la vía de salida natural: el compartir empáticamente en grupo; en este contexto el grupo se convierte en una importante herramienta terapéutica ${ }^{(12)}$.

Un aspecto muy relevante en la adolescencia es el cuerpo. Hemos subrayado cómo en esta etapa del desarrollo humano los cambios corporales y sexuales representan un aspecto importante y central de la vida. Sentirse adecuado dependerá de la opinión que se piensa que los demás tendrán de uno. El cáncer y las diferentes modalidades terapéuticas empleadas para controlarlo, producen en muchos casos alteraciones en la imagen corporal del paciente. Alopecia, cambios en las uñas, alteraciones dermatológicas, cicatrices, amputaciones, cambios en el peso, etc. hacen que el paciente se vea obligado a aceptar un cuerpo que ahora es diferente al que poseía antes del diagnóstico del cáncer.

La imagen corporal incluye percepción, pensamientos y sentimientos, y hace referencia a la imagen que formamos en nuestra mente de nuestro propio cuerpo, es decir, de cómo lo percibimos y de cómo lo sentimos.

Los cambios físicos provocados por la enfermedad y por los tratamientos, tanto que sean temporales o que sean permanentes, suponen un rol fundamental en la experiencia del niño. La nueva imagen corporal tiene que ser aceptada y se evidencia una reacción de duelo como la que ya se había experimentado en la fase del diagnostico. Los adolescentes enfermos de cáncer deben conseguir integrar en el complejo proceso de definición de la identidad personal una imagen del cuerpo alterada por la enfermedad: es una tarea emotiva, además de cognitiva, en la cual es fundamental que los signos de la enfermedad no sean identificados con los símbolos principales de la nueva identidad.

Para favorecer este proceso de integración la Fundación Aladina actúa en dos frentes, si por un lado colabora económicamente en la adquisición de pelucas; por el otro ayuda a normalizar la relación con el cuerpo.

La alopecia es un aspecto que genera mucho malestar porque es el indicador del cáncer frente a los demás ${ }^{(13)}$, por este motivo en el 2009 Aladina colabora con la Asociación española de Estética Reparadora Integral (AEERI) donando pelucas de pelo natural a los adolescentes que lo necesiten para favorecer la adaptación a la nueva situación.

Lo que hemos notado con los años y teniendo ya un numero de donaciones superior a 50 pelucas, mayormente para chicas, es que la posibilidad de disponer de una solución antes de que tenga lugar la caída del pelo, hace bajar notablemente los niveles de ansiedad y de impotencia, favoreciendo la adherencia a los tratamientos y la percepción de control sobre la enfermedad.

También iniciativas como los talleres de maquillaje han sido de gran ayuda para recuperar una buena relación con la imagen corporal; y los talleres de salsa para fomentar la movilización del cuerpo, como todos los juegos que implican el movimiento.

Además no hay que olvidar la importancia del contacto físico: a través de esto llegan mensajes de reafirmación sobre el sentido de intocabilidad y la vergüenza del propio cuerpo machacado por la enfermedad y los tratamientos y se da la prueba inconmutable que no están solos ${ }^{(14)}$.

\section{CONCLUSIONES}

La finalidad de la Fundación Aladina, en todas las iniciativas que lleva a cabo, es reducir el impacto traumático de la experien- 
cia de la enfermedad, siendo concientes de las necesidades psicológicas y emocionales de los chicos y de sus familias. Intentamos mejorar el entorno físico y apoyarlos emocionalmente para que puedan sobrellevar el impacto del cáncer y sus consecuencias. Acompañamos al adolescente en su proceso sin banalizarlo ni ejerciendo presión, tratándole con la mayor naturalidad posible. Lo que pretendemos es fomentar el desarrollo normal de los niños y de los adolescentes haciendo que el cáncer no sea, más de lo que ya es, un trauma.

El mensaje que llevamos como bandera es que la enfermedad es una etapa de la vida y no debe significar un bloqueo. El estar enfermo no debería ser motivo suficiente para perder la alegría y la ilusión de vivir.

Todo el equipo de la Fundación se mueve por la convicción de que cuando se crea un contacto genuino con los chicos, el equilibrio perdido se reencuentra y se renuevan las energías. El intercambio de experiencias y de motivación hace que tanto los voluntarios como los chicos crezcan y encuentren nuevos caminos para solucionar conflictos y bloqueos. Poniendo palabras a lo que está ocurriendo, a los pensamientos y a las emociones, facilitamos la contención de los miedos que impiden avanzar en el proceso de adaptación.

Cada día cuando cerramos la sala somos conscientes de que hemos contribuido a regalar diversión, ofrecer un apoyo y, sobretodo, que un día más en el hospital ya ha pasado. Los adolescentes vuelven a las habitaciones con una sonrisa y las ganas de seguir mas y mejor al día siguiente. Esta es la esencia de la Fundación Aladina.

Partiendo de la convicción de que el ser humano es una unión indisoluble de mente y cuerpo, el estado de ánimo es un aliado fundamental y necesario para que el físico responda. No está en nuestras manos manejar los tratamientos físicos así que queremos poner nuestro granito de arena en favorecer el bienestar emocional de nuestros niños y adolescentes porque "una sonrisa hace magia".

\section{REFERENCIAS BIBLIOGRÁFICAS}

1. Biondi G., Valenzi C. Problemi ed ipotesi operative di un intervento di psicologia sociale all'interno di un ospedale.; Atti e memorie dell'Accademia di storia e dell'arte sanitaria, 1984 n.1/2: 183-8.

2. McPhilips $H$, Gallaher $M$, Koepsell $T$. Children hospitalized early and increased risk for future serious injury. Inj Prev $2001 ; 7: 150-4$.

3. Whaley L, Wong D, Tratado de enfermería pediátrica. McGraw-Hill, 1988.

4. Kubler Ross E. La rueda de la vida. Barcelona: Ediciones B Grupo Z, 2003.

5. Muñiz M, Santos H, Kotliarenco MA, Suárez EN, Infante, F, Grotberg E. Manual de identificación y promoción de la resiliencia en niños y adolescentes. Washington: Fundación WK Kellog, 1998.

6. Die Trill, M.: Niños y adolescentes con cáncer. Aspectos psicológicos. Jano 1987; XXX111:35-41.

7. Guarino A. Psiconcologia dell'età evolutiva. La psicologia nelle cure dei bambini malati di cancro. Trento: Centro Studi Erickson, 2006.

8. Scarponi, D. Dolore procedurale e sofferenza in oncoematologia pediatrica. La giocoterapia come supporto psicologico. Giornale Italiano di Psico-Oncologia 2007; 9:1. Doi: 10.1400/164773.

9. Biondi G., Il bambino malato e il non rispetto dei suoi diritti. Riv. Scient di Psico 2002;1:25-35

10. Barrera ME, Rykov MH, Doyle SL. The effects of interactive music therapy on hospitalized children with cancer: a pilot study. Psychooncology 2002; 11:379-88. Doi: 10.1002/pon.589

11. Die Trill M. El niño y adolescente con cáncer. En Die Trill, editor. Psico-oncología. Madrid: ADES Ediciones, 2003.p.86-101 
12. Cornejo, L. Manual de terapia infantil gestaltica. Bilbao: Desclee de Brower, 1996.

13. Williamson $H_{\text {., Harcourt }} \mathrm{D}$, Halliwell E, Frith $H$, Wallace $M$. adolescents' and parents' experiences of managing the psychosocial impact of appearance change during cancer treatment J Pediatr Oncol Nurs 2010;27:168-75. Doi: 10.1177/1043454209357923

14. Sourkes BM. II tempo tra le braccia. L'esperienza psicologia del bambino affetto da tumore. Milano: Raffaello Cortina, 1999. 